\title{
ARTICLE
}

Received 2 Jul 2014 | Accepted 12 May 2015 | Published 23 Jun $2015 \quad$ DOl: 10.1038/ncomms8498

\section{Maxwell's demon in biochemical signal transduction with feedback loop}

Sosuke Ito ${ }^{1, \dagger} \&$ Takahiro Sagawa ${ }^{2, \dagger}$

Signal transduction in living cells is vital to maintain life itself, where information transfer in noisy environment plays a significant role. In a rather different context, the recent intensive research on 'Maxwell's demon'-a feedback controller that utilizes information of individual molecules-have led to a unified theory of information and thermodynamics. Here we combine these two streams of research, and show that the second law of thermodynamics with information reveals the fundamental limit of the robustness of signal transduction against environmental fluctuations. Especially, we find that the degree of robustness is quantitatively characterized by an informational quantity called transfer entropy. Our information-thermodynamic approach is applicable to biological communication inside cells, in which there is no explicit channel coding in contrast to artificial communication. Our result could open up a novel biophysical approach to understand information processing in living systems on the basis of the fundamental information-thermodynamics link.

\footnotetext{
${ }^{1}$ Department of Physics, The University of Tokyo, Tokyo 113-0033, Japan. ${ }^{2}$ Department of Basic Science, The University of Tokyo, Tokyo 153-8902, Japan. $\dagger$ Present addresses: Department of Physics, Tokyo Institute of Technology, Tokyo 152-8551, Japan (S.I.); Department of Applied Physics, The University of Tokyo, Tokyo 113-8656, Japan (T.S.). Correspondence and requests for materials should be addressed to S.I. (email: sosuke@stat.phys.titech.ac.jp).
} 
A crucial feature of biological signal transduction lies in the fact that it works in noisy environment ${ }^{1-3}$. To understand its mechanism, signal transduction has been modelled as noisy information processing ${ }^{4-11}$. For example, signal transduction of bacterial chemotaxis of Escherichia coli (E. coli) has been investigated as a simple model organism for sensory adaptation ${ }^{12-16}$. A crucial ingredient of $E$. coli chemotaxis is a feedback loop, which enhances the robustness of the signal transduction against environmental noise.

The information transmission inside the feedback loop can be quantified by the transfer entropy, which was originally introduced in the context of time series analysis ${ }^{17}$, and has been studied in electrophysiological systems ${ }^{18}$, chemical processes $^{19}$ and artificial sensorimotors ${ }^{20}$. The transfer entropy is the conditional mutual information representing the directed information flow, and gives an upper bound of the redundancy of the channel coding in an artificial communication channel with a feedback loop ${ }^{21}$; this is a fundamental consequence of Shannon's second theorem 22,23 . However, as there is not any explicit channel coding inside living cells, the role of the transfer entropy in biological communication has not been fully understood.

The transfer entropy also plays a significant role in thermodynamics ${ }^{24}$. Historically, the connection between thermodynamics and information was first discussed in the thought experiment of 'Maxwell's demon' in the nineteenth century $25-27$, where the demon is regarded as a feedback controller. In the recent progress on this problem in light of modern non-equilibrium statistical physics ${ }^{28,29}$, a universal and quantitative theory of thermodynamics feedback control has been developed, leading to the field of information thermodynamics ${ }^{24,30-48}$. Information thermodynamics reveals a generalization of the second law of thermodynamics, which implies that the entropy production of a target system is bounded by the transfer entropy from the target system to the outside world ${ }^{24}$.

In this article, we apply the generalized second law to establish the quantitative relationship between the transfer entropy and the robustness of adaptive signal transduction against noise. We show that the transfer entropy gives the fundamental upper bound of the robustness, elucidating an analogy between information thermodynamics and the Shannon's information theory ${ }^{22,23}$. We numerically studied the information-thermodynamics efficiency of the signal transduction of $E$. coli chemotaxis, and found that the signal transduction of $E$. coli chemotaxis is efficient as an information-thermodynamic device, even when it is highly dissipative as a conventional heat engine.

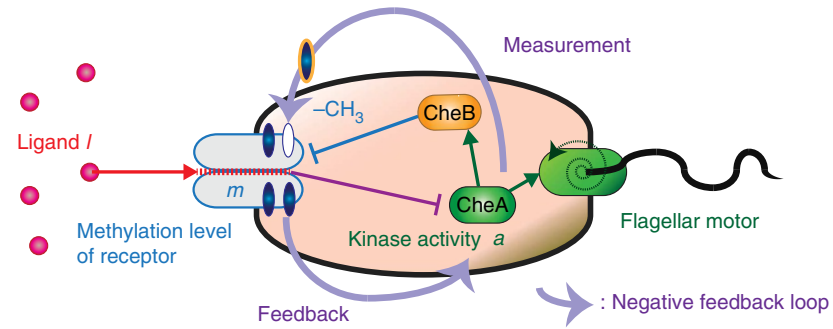

Figure 1 | Schematic of adaptive signal transduction of $E$. coli bacterial chemotaxis. Kinase activity a (green) activates a flagellar motor to move E. coli towards a direction of the higher ligand density I (red) by using the information stored in methylation level $m$ (blue). CheA is the histidine kinase related to the flagellar motor, and the response regulator $C h e B$, activated by CheA, removes methyl groups from the receptor. The methylation level $m$ plays a similar role to the memory of Maxwell's demon 8,24 , which reduces the effect of the environmental noise on the target system $a$; the negative feedback loop (purple arrows) counteracts the influence of ligand binding.

\section{Results}

Model. The main components of E. coli chemotaxis are the ligand density change $l$, the kinase activity $a$ and the methylation level $m$ of the receptor (Fig. 1). A feedback loop exists between $a$ and $m$, which reduces the environmental noise in the signal transduction pathway from $l$ to $a$ (ref. 49). Let $l_{t}, a_{t}$ and $m_{t}$ be the values of these quantities at time $t$. They obey stochastic dynamics due to the noise, and are described by the the following coupled Langevin equations ${ }^{7,14,16}$ :

$$
\begin{aligned}
& \dot{a}_{t}=-\frac{1}{\tau^{a}}\left[a_{t}-\bar{a}_{t}\left(m_{t}, l_{t}\right)\right]+\xi_{t}^{a}, \\
& \dot{m}_{t}=-\frac{1}{\tau^{m}} a_{t}+\xi_{t}^{m},
\end{aligned}
$$

where $\bar{a}_{t}\left(m_{t}, l_{t}\right)$ is the stationary value of the kinase activity under the instantaneous values of the methylation level $m_{t}$ and the ligand signal $l_{t}$. In the case of $E$. coli chemotaxis, we can approximate $\bar{a}_{t}\left(m_{t}, l_{t}\right)$ as $\alpha m_{t}-\beta l_{t}$, by linearizing it around the steady-state value ${ }^{7,14}$. $\xi_{t}^{x}(x=a, m)$ is the white Gaussian noise with $\left\langle\xi_{t}^{x}\right\rangle=0$ and $\left\langle\xi_{t}^{x} \xi_{t^{\prime}}^{x^{\prime}}\right\rangle=2 T_{t}^{x} \delta_{x x^{\prime}} \delta\left(t-t^{\prime}\right)$, where $\langle\cdots\rangle$ describes the ensemble average. $T_{t}^{x}$ describes the intensity of the environmental noise at time $t$, which is not necessarily thermal inside cells. The noise intensity $T_{t}^{a}$ characterizes the ligand fluctuation. The time constants satisfy $\tau^{m} \gg \tau^{a}>0$, which implies that the relaxation of $a$ to $\bar{a}_{t}$ is much faster than that of $m$.

The mechanism of adaptation in this model is as follows (Fig. 2; refs 14,16). Suppose that the system is initially in a stationary state with $l_{t}=0$ and $a_{t}=\bar{a}_{t}\left(m_{t}, 0\right)=0$ at time $t<0$, and $l_{t}$ suddenly changes from 0 to 1 at time $t=0$ as a step function. Then, $a_{t}$ rapidly equilibrates to $\bar{a}_{t}\left(m_{t}, 1\right)$ so that the difference $a_{t}-\bar{a}_{t}$ becomes small. The difference $a_{t}-\bar{a}_{t}$ plays an important role, which characterizes the level of adaptation. Next, $m_{t}$ gradually changes to satisfy $\bar{a}_{t}\left(m_{t}, 1\right)=0$, and thus $a_{t}$ returns to 0 , where $a_{t}-\bar{a}_{t}$ remains small.

Robustness against environmental noise. We introduce a key quantity that characterizes the robustness of adaptation, which is defined as the difference between the intensity of the ligand noise $T_{t}^{a}$ and the mean square error of the level of adaptation $\left\langle\left(a_{t}-\bar{a}_{t}\right)^{2}\right\rangle:$

$$
J_{t}^{a}:=\frac{1}{\tau^{a}}\left[T_{t}^{a}-\frac{1}{\tau^{a}}\left\langle\left(a_{t}-\bar{a}_{t}\right)^{2}\right\rangle\right] .
$$

The larger $J_{t}^{a}$ is, the more robust the signal transduction is against the environmental noise. In the case of thermodynamics, $J_{t}^{a}$ corresponds to the heat absorption in $a$ and characterizes the

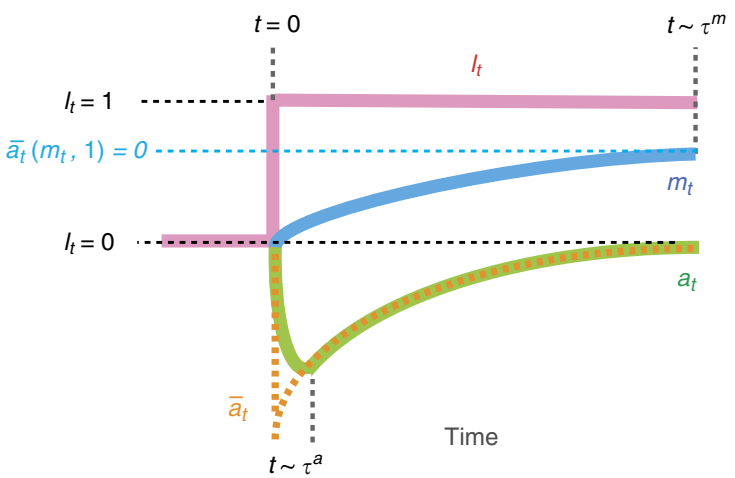

Figure 2 | Typical dynamics of adaptation with the ensemble average. Suppose that $l_{t}$ changes as a step function (red solid line). Then, $a_{t}$ suddenly responds (green solid line), followed by the gradual response of $m_{t}$ (blue solid line). The adaptation is achieved by the relaxation of $a_{t}$ to $\bar{a}_{t}$ (orange dashed line). The methylation level $m_{t}$ gradually changes to $\bar{a}_{t}\left(m_{t}, 1\right)=0$ (blue dashed line). 
violation of the fluctuation-dissipation theorem ${ }^{28}$. Since the environmental noise is not necessarily thermal in the present situation, $J_{t}^{a}$ is not exactly the same as the heat, but is a biophysical quantity that characterizes the robustness of adaptation against the environmental noise.

Information flow. We here discuss the quantitative definition of the transfer entropy ${ }^{17}$. The transfer entropy from $a$ to $m$ at time $t$ is defined as the conditional mutual information between $a_{t}$ and $m_{t+\mathrm{d} t}$ under the condition of $m_{t}$ :

$$
\mathrm{d} I_{t}^{\mathrm{tr}}:=\int \mathrm{d} m_{t+\mathrm{d} t} \mathrm{~d} a_{t} \mathrm{~d} m_{t} p\left[m_{t+\mathrm{d} t}, a_{t}, m_{t}\right] \ln \frac{p\left[m_{t+\mathrm{d} t} \mid a_{t}, m_{t}\right]}{p\left[m_{t+\mathrm{d} t} \mid m_{t}\right]},
$$

where $p\left[m_{t+\mathrm{d} t}, a_{t}, m_{t}\right]$ is the joint probability distribution of $\left(m_{t+\mathrm{d} t}, a_{t}, m_{t}\right)$, and $p\left[m_{t+\mathrm{d} t} \mid a_{t}, m_{t}\right]$ is the probability distribution of $m_{t+\mathrm{d} t}$ under the condition of $\left(a_{t}, m_{t}\right)$. The transfer entropy characterizes the directed information flow from $a$ to $m$ during an infinitesimal time interval $\mathrm{d} t$ (refs 17,50), which quantifies a causal influence between them ${ }^{51,52}$. From the non-negativity of the conditional mutual information ${ }^{23}$, that of the transfer entropy follows: $\mathrm{d} I_{t}^{\mathrm{tr}} \geq 0$.

Second law of information thermodynamics. We now consider the second law of information thermodynamics, which characterizes the entropy change in a subsystem in terms of the information flow (Fig. 3). In the case of equation (1), the generalized second law is given as follows (see also Methods section):

$$
\mathrm{d} I_{t}^{\mathrm{tr}}+\mathrm{d} S_{t}^{a \mid m} \geq \frac{J_{t}^{a}}{T_{t}^{a}} \mathrm{~d} t
$$

Here, $\mathrm{d} S_{t}^{a \mid m}$ is the conditional Shannon entropy change defined as $\mathrm{d} S_{t}^{a \mid m}:=S\left[a_{t+\mathrm{d} t} \mid m_{t+\mathrm{d} t} t-S\left[a_{t} \mid m_{t}\right]\right.$ with $S\left[a_{t} \mid m_{t}\right]:=$ $-\int \mathrm{d} a_{t} \mathrm{~d} m_{t} p\left[a_{t}, m_{t}\right] \ln p\left[a_{t} \mid m_{t}\right]$, which vanishes in the stationary state. The transfer entropy $\mathrm{d} I_{t}^{\text {tr }}$ on the left-hand side of a

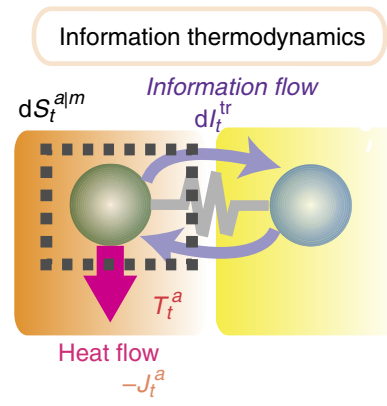

b

Conventional thermodynamics

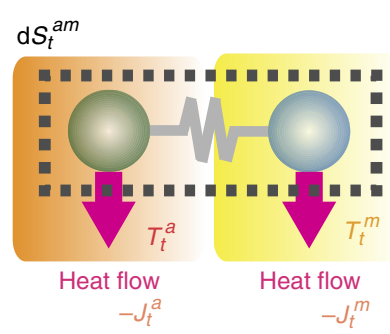

Figure 3 | Schematics of information thermodynamics and conventional thermodynamics. A green (blue) circle indicates subsystem $a(m)$ and a grey polygonal line indicates their interaction. (a) The second law of information thermodynamics characterizes the entropy change in a subsystem in terms of the information flow between the subsystem and the outside world (that is, $\Xi_{t}^{\text {info }}:=\left.\mathrm{d}\right|_{t} ^{t r}+\mathrm{d} S_{t}^{a \mid m} \geq-\int_{t}^{a} \mathrm{~d} t / T_{t}^{a}$ ). The informationthermodynamics picture concerns the entropy change inside the dashed square that only includes subsystem a. (b) the conventional second law of thermodynamics states that the entropy change in a subsystem is compensated for by the entropy change in the outside world (that is, $\left.\Xi_{t}^{\mathrm{SL}}:=-J_{t}^{m} \mathrm{~d} t / T_{t}^{m}+\mathrm{d} S_{t}^{a m} \geq J_{t}^{a} \mathrm{~d} t / T_{t}^{a}\right)$. The conventional thermodynamics picture concerns the entropy change inside the dashed square, which includes the entire systems $a$ and $m$. As explicitly shown in this paper, information thermodynamics gives a tighter bound of the robustness $\int_{t}^{a}$ in the biochemical signal transduction of $E$. coli chemotaxis. equation (4) shows the significant role of the feedback loop, implying that the robustness of adaptation can be enhanced against the environmental noise by the feedback using information. This is analogous to the central feature of Maxwell's demon.

To further clarify the meaning of inequality (equation (4)), we focus on the case of the stationary state. If there was no feedback loop between $m$ and $a$, then the second law reduces to $\left\langle\left(a_{t}-\bar{a}_{t}\right)^{2}\right\rangle \geq \tau^{a} T_{t}^{a}$, which, as naturally expected, implies that the fluctuation of the signal transduction is bounded by the intensity of the environmental noise. In contrast, in the presence of a feedback loop, $\left\langle\left(a_{t}-\bar{a}_{t}\right)^{2}\right\rangle$ can be smaller than $\tau^{a} T_{t}^{a}$ owing to the transfer entropy $\mathrm{d} I_{t}^{\mathrm{tr}}$ in the feedback loop:

$$
\left\langle\left(a_{t}-\bar{a}_{t}\right)^{2}\right\rangle \geq \tau^{a} T_{t}^{a}\left[1-\frac{\mathrm{d} I_{t}^{\mathrm{tr}}}{\mathrm{d} t} \tau^{a}\right] .
$$

This inequality clarifies the role of the transfer entropy in biochemical signal transduction; the transfer entropy characterizes an upper bound of the robustness of the signal transduction in the biochemical network. The equality in equation (5) is achieved in the limit of $\alpha \rightarrow 0$ and $\tau^{a} / \tau^{m} \rightarrow 0$ for the linear case with $\bar{a}_{t}\left(m_{t}, l_{t}\right)=\alpha m_{t}-\beta l_{t}$ (Supplementary Note 1). The latter limit means that $a$ relaxes infinitely fast and the process is quasi-static (that is, reversible) in terms of $a$. This is analogous to the fact that Maxwell's demon can achieve the maximum thermodynamics gain in reversible processes ${ }^{35}$. In general, the information-thermodynamic bound becomes tight if $\alpha$ and $\tau^{m} / \tau^{a}$ are both small. The realistic parameters of the bacterial chemotaxis are given by $\alpha \simeq 3$ and $\tau^{a} / \tau^{m} \simeq 0.1$ (refs $7,14,16$ ), and therefore the real adaptation process is accompanied by a finite amount of information-thermodynamics dissipation.

Our model of chemotaxis has the same mathematical structure as the feedback cooling of a colloidal particle by Maxwell's demon $^{36,38,42,47}$, where the feedback cooling is analogous to the noise filtering in the sensory adaptation ${ }^{49}$. This analogy is a central idea of our study; the information-thermodynamic inequalities (equation (5) in our case) characterize the robustness of adaptation as well as the performance of feedback cooling.

Numerical result. We consider the second law (equation (4)) in non-stationary dynamics, and numerically demonstrate the power of this inequality. Figure 4 shows $J_{t}^{a} \mathrm{~d} t / T_{t}^{a}$ and

$$
\Xi_{t}^{\text {info }}:=\mathrm{d} I_{t}^{\mathrm{tr}}+\mathrm{d} S_{t}^{a \mid m}
$$

in six different types of dynamics of adaptation, where the ligand signal is given by a step function (Fig. 4a), a sinusoidal function (Fig. 4b), a linear function (Fig. 4c), an exponential decay (Fig. 4d), a square wave (Fig. 4e) and a triangle wave (Fig. 4f). These results confirm that $\Xi_{t}^{\text {info }}$ gives a tight bound of $J_{t}^{a}$, implying that the transfer entropy characterizes the robustness well. In Fig. $4 \mathrm{~b}, f$, the robustness $J_{t}^{a} \mathrm{~d} t / T_{t}^{a}$ is nearly equal to the information-thermodynamics bound $\Xi_{t}^{\text {info }}$ when the signal and noise are decreasing or increasing rapidly (for example, $t \simeq 0.008$ and $t=0.012$ in Fig. 4f)

Conventional second law of thermodynamics. For the purpose of comparison, we next consider another upper bound of the robustness, which is given by the conventional second law of thermodynamics without information. We define the heat absorption by $m$ as $J_{t}^{m}:=-\left\langle a_{t}^{2}\right\rangle /\left(\tau^{m}\right)^{2}$, and the Shannon entropy change in the total system as $\mathrm{d} S_{t}^{a m}:=S\left[a_{t+\mathrm{d} t}, m_{t+\mathrm{d} t}\right]-S\left[a_{t}, m_{t}\right]$ with $S\left[a_{t}, m_{t}\right]:=-\int \mathrm{d} a_{t} \mathrm{~d} m_{t} p\left[a_{t}, m_{t}\right] \ln p\left[a_{t}, m_{t}\right]$, which vanishes 
a
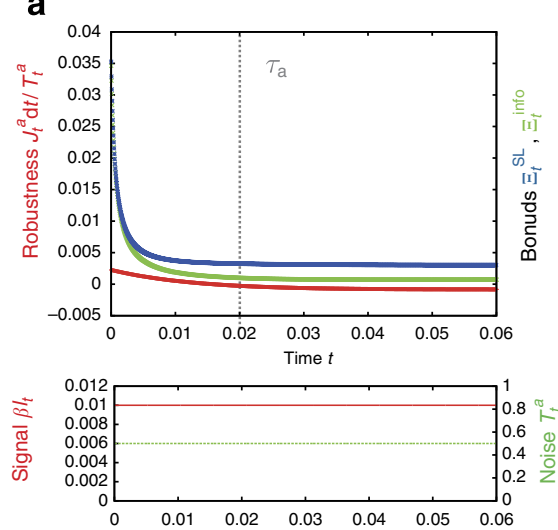

d
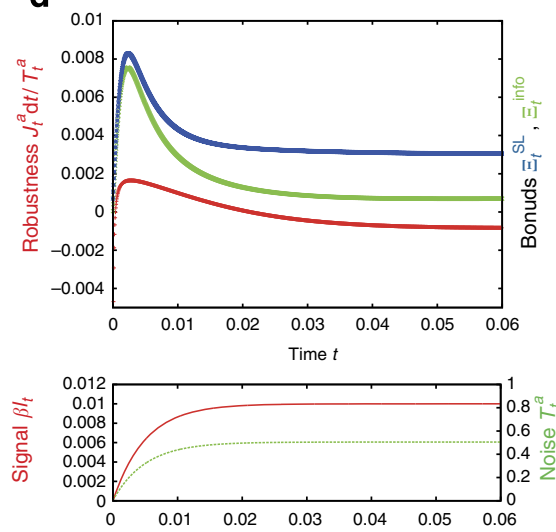

b
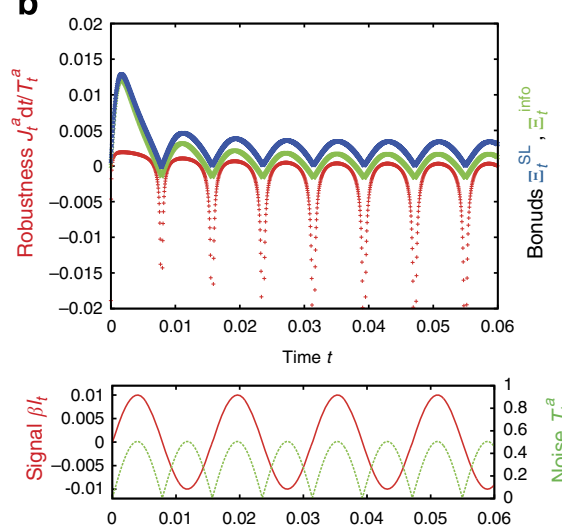

$\mathbf{e}$
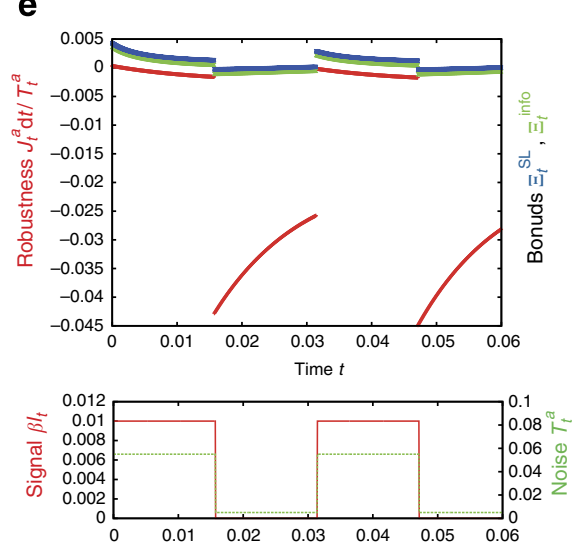

C
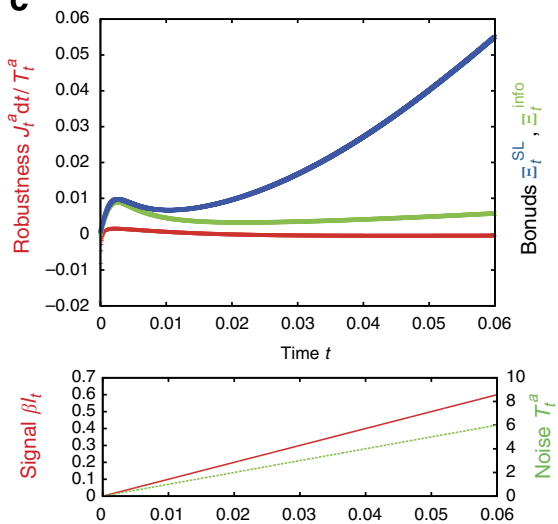

f
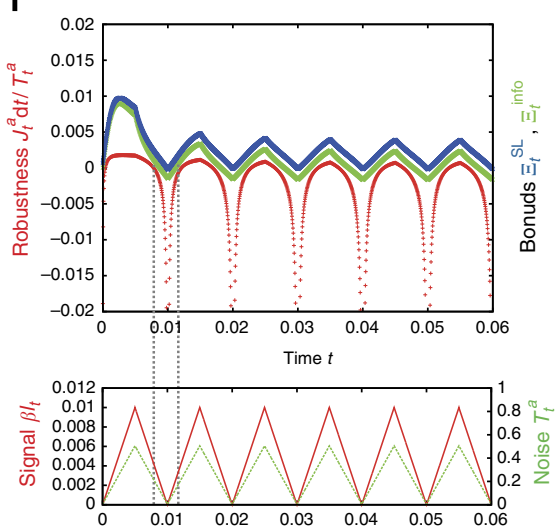

Figure 4 | Numerical results of the information-thermodynamics bound on the robustness. We compare the robustness ja (red line), the informationthermodynamic bound $\Xi_{t}^{\text {info }}$ (green line) and the conventional thermodynamic bound $\Xi_{t}^{\text {SL }}$ (blue line). The initial condition is the stationary state with $\bar{a}_{t}=\alpha m_{t}-\beta l_{t}$, fixed ligand signal $\beta l_{t}=0$, and noise intensity $T^{a}=0.005$. We numerically confirmed that $\Xi_{t}^{\mathrm{SL}} \geq \Xi_{t}^{\text {info }} \geq J_{t}^{a} \mathrm{~d} t / T_{t}^{a}$ holds for the six transition processes. These results imply that, for the signal transduction model, the information-thermodynamic bound is tighter than the conventional thermodynamic bound. The parameters are chosen as $\tau^{a}=0.02, \tau^{m}=0.2, \alpha=2.7$ and $T_{t}^{m}=0.005$ to be consistent with the real parameters of $E$. coli bacterial chemotaxis $7,14,16$. We discuss the six different types of input signals $\beta l_{t}$ (red solid line) and noises $T_{t}^{a}$ (green dashed line). (a) Step function: $\beta I_{t}=0.01$ and $T_{t}^{a}=0.5$ for $t>0$. (b) Sinusoidal function: $\beta l_{t}=0.01 \sin (400 t)$ and $T_{t}^{a}=0.5|\sin (400 t)|+0.005$ for $t>0$. (c) Linear function: $\beta I_{t}=10 t$ and $T_{t}^{a}=100 t+0.005$ for $t>0$. (d) Exponential decay: $\beta L_{t}=0.01[1-\exp (-200 t)]$ and $T_{t}^{a}=0.5[1-\exp (-200 t)]+0.005$ for $t>0$. (e) Square wave: $\beta l_{t}=0.01[1+\lfloor\sin (200 t)\rfloor]$ and $T_{t}^{a}=0.05[1+\lfloor\sin (200 t)\rfloor]+0.005$ for $t>0$, where $\lfloor\ldots\rfloor$ denotes the floor function. (f) Triangle wave: $\beta l_{t}=0.01|2(100 t-\lfloor 100 t+0.5\rfloor)|$ and $T_{t}^{a}=0.5|2(100 t-\lfloor 100 t+0.5\rfloor)|+0.005$ for $t>0$.

in the stationary state. We can then show that

$$
\Xi_{t}^{\mathrm{SL}}:=-\frac{J_{t}^{m}}{T_{t}^{m}} \mathrm{~d} t+\mathrm{d} S_{t}^{a m}
$$

is an upper bound of $J_{t}^{a} \mathrm{~d} t / T_{t}^{a}$, as a straightforward consequence of the conventional second law of thermodynamics of the total system of $a$ and $m$ (refs 28,29). The conventional second law implies that the dissipation in $m$ should compensate for that in $a$ (Fig. 3). Figure 4 shows $J_{t}^{a} \mathrm{~d} t / T_{t}^{a}$ along with $\Xi_{t}^{\text {info }}$ and $\Xi_{t}^{\mathrm{SL}}$. Remarkably, information-thermodynamic bound $\Xi_{t}^{\text {info }}$ gives a tighter bound of $J_{t}^{a}$ than the conventional thermodynamics bound $\Xi_{t}^{\mathrm{SL}}$ such that

$$
\Xi_{t}^{\mathrm{SL}} \geq \Xi_{t}^{\mathrm{info}} \geq \frac{J_{t}^{a}}{T_{t}^{a}} \mathrm{~d} t
$$

for every non-stationary dynamics shown in Fig. 4. Moreover, we can analytically show inequalities (equation (8)) in the stationary state (Supplementary Note 4).

To compare the information-thermodynamic bound and the conventional thermodynamics one more quantitatively, we introduce an information-thermodynamic figure of merit based on the inequalities (equation (8)):

$$
\chi:=1-\frac{\Xi_{t}^{\text {info }}-J_{t}^{a} \mathrm{~d} t / T_{t}^{a}}{\Xi_{t}^{\mathrm{SL}}-J_{t}^{a} \mathrm{~d} t / T_{t}^{a}}
$$

where the second term on the right-hand side is given by the ratio between the information-thermodynamic dissipation $\Xi_{t}^{\text {info }}-J_{t}^{a} \mathrm{~d} t / T_{t}^{a}$ and the entire thermodynamic dissipation $\Xi_{t}^{\mathrm{SL}}-J_{t}^{a} \mathrm{~d} t / T_{t}^{a}$. This quantity satisfies $0 \leq \chi \leq 1$, and $\chi \simeq 1 \quad(\chi \simeq 0)$ means that information-thermodynamic bound is much tighter (a little tighter) compared with the conventional thermodynamic bound. We numerically calculated $\chi$ in the aforementioned six types of dynamics of adaptation (Supplementary Figs 1-6). In the case of a linear function (Supplementary Fig. 3), we found that $\chi$ increases in time $t$ and approaches to $\chi \simeq 1$. In this case, the signal transduction of E. coli chemotaxis is highly dissipative as a thermodynamic engine, but efficient as an information transmission device.

Comparison with Shannon's theory. We here discuss the similarity and the difference between our result and the Shannon's information theory (refs 22,23; Fig. 5). The Shannon's second 
a

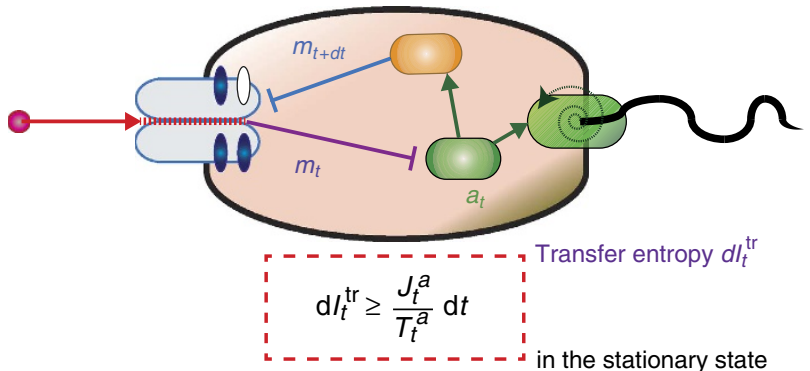

b

Achievable information rate

(Robustness of information transmission against noise) $R$

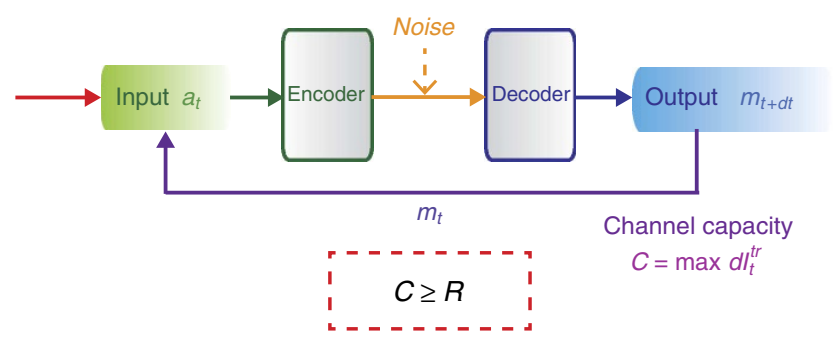

Figure 5 | Analogy and difference between our approach and Shannon's information theory. (a) Information thermodynamics for biochemical signal transduction. The robustness $J_{t}^{a}$ is bounded by the transfer entropy $\mathrm{d}_{t}^{\mathrm{tr}}$ in the stationary states, which is a consequence of the second law of information thermodynamics. (b) Information theory for artificial communication. The archivable information rate $R$, given by the redundancy of the channel coding, is bounded by the channel capacity $C=\max \mathrm{d}_{t}^{\text {tr }}$, which is a consequence of the Shannon's second theorem. If the noise is Gaussian as is the case for the E. coli chemotaxis, both of the transfer entropy and the channel capacity are given by the power-to-noise ratio $C=\mathrm{d}_{t}^{t r}=(2)^{-1} \ln \left(1+\mathrm{d} P_{t} / N_{t}\right)$, under the condition that the initial distribution is Gaussian (see Methods section).

theorem (that is, the noisy-channel coding theorem) states that an upper bound of achievable information rate $R$ is given by the channel capacity $C$ such that $C \geq R$. The channel capacity $C$ is defined as the maximum value of the mutual information with finite power, where the mutual information can be replaced by the transfer entropy $\mathrm{d} I_{t}^{\mathrm{tr}}$ in the presence of a feedback loop ${ }^{21} . R$ describes how long bit sequence is needed for a channel coding to realize errorless communication through a noisy channel, where errorless means the coincidence between the input and output messages. Therefore, both of $J_{t}^{a}$ and $R$ characterize the robustness information transmission against noise, and bounded by the transfer entropy $\mathrm{d} I_{t}^{\mathrm{tr}}$. In this sense, there exists an analogy between the second law of thermodynamics with information and the Shannon's second theorem. In the case of biochemical signal transduction, the information-thermodynamic approach is more relevant, because there is not any explicit channel coding inside cells. Moreover, while $J_{t}^{a}$ is an experimentally measurable quantity as mentioned below ${ }^{28,29}, R$ cannot be properly defined in the absence of any artificial channel coding 23 . Therefore, $J_{t}^{a}$ is an intrinsic quantity to characterize the robustness of the information transduction inside cells.

\section{Discussion}

Our result can be experimentally validated by measuring the transfer entropy and thermodynamics quantities from the probability distribution of the amount of proteins in a biochemical system $^{5,6,9,10,46-49}$. In fact, the transfer entropy $\mathrm{d} I_{\mathrm{tr}}$ and thermodynamics quantities (that is, $\mathrm{d} S_{t}^{a \mid m}$ and $J_{t}^{a} \mathrm{~d} t / T_{t}^{a}$ ) can be obtained from the joint probability distribution of $\left(a_{t}, m_{t}, a_{t+\mathrm{d} t}, m_{t+\mathrm{d} t}\right)$. The measurement of such a joint distribution would not be far from today's experimental technique in biophysics $5,6,9,10,53-56$. Experimental measurements of $\Xi_{t}^{\text {info }}$ and $J_{t}^{a} \mathrm{~d} t / T_{t}^{a}$ would lead to a novel classification of signal transduction in terms of the thermodynamics cost of information transmission.

We note that, in ref. 16, the authors discussed that the entropy changes in two heat baths $-J_{t}^{a} / T_{t}^{a}-J_{t}^{m} / T_{t}^{m} \simeq-J_{t}^{m} / T_{t}^{m}=$ $\left\langle a_{t}^{2}\right\rangle /\left[T_{t}^{m}\left(\tau^{m}\right)^{2}\right]$ can be characterized by the accuracy of adaptation. In our study, we derived a bound for $J_{t}^{a} \mathrm{~d} t / T_{t}^{a}$ that is regarded as the robustness of signal transduction against the environmental noise. These two results capture complementary aspects of adaptation processes: accuracy and robustness.

We also note that our theory of information thermodynamics 24 can be generalized to a broad class of signal transduction networks, including a feedback loop with time delay.

\section{Methods}

The outline of the derivation of inequality (4). We here show the outline of the derivation of the information-thermodynamic inequality (equation (4); see also Supplementary Note 2 for details). The heat dissipation $J_{t}^{a} \mathrm{~d} t / T_{t}^{a}$ is given by the ratio between forward and backward path probabilities as $J_{t}^{a} \mathrm{~d} t / T_{t}^{a}=$ $\int \mathrm{d} a_{t} \mathrm{~d} a_{t+\mathrm{d} t} \mathrm{~d} m_{t} p\left[a_{t}, a_{t+\mathrm{d} t}, m_{t}\right] \ln \left[p_{B}\left[a_{t} \mid a_{t+\mathrm{d} t}, m_{t}\right] / p\left[a_{t+\mathrm{d} t} \mid a_{t}, m_{t}\right]\right]$ (refs 24,28,29), where the backward path probability $p_{B}\left[a_{t} \mid a_{t+\mathrm{d} t}, m_{t}\right]:=\mathcal{G}\left(a_{t} ; a_{t+\mathrm{d} t} ; m_{t}\right)$ can be calculated from the forward path probability $p\left[a_{t+\mathrm{d} t} \mid a_{t}, m_{t}\right]=: \mathcal{G}\left(a_{t+\mathrm{d} t} ; a_{t} ; m_{t}\right)$. Thus, the difference $\mathrm{d} I_{t}^{\mathrm{tr}}+\mathrm{d} S_{t}^{a \mid m}-J_{t}^{a} \mathrm{~d} t / T_{t}^{a}$ is given by the Kullback-Libler divergence ${ }^{23}$. From its non-negativity ${ }^{23}$, we have $\mathrm{d} I_{t}^{\mathrm{tr}}+\mathrm{d} S_{t}^{a \mid m} \geq J_{t}^{a} \mathrm{~d} t / T_{t}^{a}$. This inequality can be derived from the general inequality of information thermodynamics $^{24}$ (see Supplementary Note 3 and Supplementary Fig. 7). As discussed in Supplementary Note 3, this inequality gives a weaker bound of the entropy production.

The analytical expression of the transfer entropy. In the case of $E$. coli chemotaxis, we have $\bar{a}_{t}=\alpha m_{t}-\beta l_{t}$, and equation (1) become linear. In this situation, if the initial distribution is Gaussian, we analytically obtain the transfer entropy up to the order of $\mathrm{d} t$ (Supplementary Note 4 ): $\mathrm{d} I_{t}^{\mathrm{tr}}=(2)^{-1} \ln \left(1+\mathrm{d} P_{t} / N_{t}\right)$, where $N_{t}:=2 T_{t}^{m}$ describes the intensity of the environmental noise, and $\mathrm{d} P_{t}:=$ $\left[1-\left(\rho_{t}^{a m}\right)^{2}\right] V_{t}^{a} \mathrm{~d} t /\left(\tau^{m}\right)^{2}$ describes the intensity of the signal from $a$ to $m$ per unit time with $V_{t}^{x}:=\left\langle x_{t}^{2}\right\rangle-\left\langle x_{t}\right\rangle^{2}$, and $\rho_{t}^{a m}:=\left[\left\langle a_{t} m_{t}\right\rangle-\left\langle a_{t}\right\rangle\left\langle m_{t}\right\rangle\right] /\left(V_{t}^{a} V_{t}^{m}\right)^{1 / 2}$. We note that $\mathrm{d} I_{\text {tr }}$ for the Gaussian case is greater than that of the non-Gaussian case, if $V_{t}^{x}$ and $\rho_{t}^{a m}$ are the same $e^{23}$. We also note that the above analytical expression of $\mathrm{d} I_{t}^{\mathrm{tr}}$ is the same form as the Shannon-Hartley theorem ${ }^{23}$.

\section{References}

1. Phillips, R., Kondev, J. \& Theriot, J. Physical Biology of the Cell (Garland Science, 2009)

2. Korobkova, E., Emonet, T., Vilar, J. M., Shimizu, T. S. \& Cluzel, P. From molecular noise to behavioural variability in a single bacterium. Nature 428, 574-578 (2004).

3. Lestas, I., Vinnicombe, G. \& Paulsson, J. Fundamental limits on the suppression of molecular fluctuations. Nature 467, 174-178 (2010).

4. Andrews, B. W. \& Iglesias, P. A. An information-theoretic characterization of the optimal gradient sensing response of cells. PLoS Comput. Biol. 3, e153 (2007).

5. Skerker, J. M. et al. Rewiring the specificity of two-component signal transduction systems. Cell 133, 1043-1054 (2008).

6. Mehta, P., Goyal, S., Long, T., Bassler, B. L. \& Wingreen, N. S. Information processing and signal integration in bacterial quorum sensing. Mol. Syst. Biol. 5, 325 (2009).

7. Tostevin, F. \& ten Wolde, P. R. Mutual information between input and output trajectories of biochemical networks. Phys. Rev. Lett. 102, 218101 (2009).

8. Tu, Y. The nonequilibrium mechanism for ultrasensitivity in a biological switch: Sensing by Maxwell's demons. Proc. Natl Acad. Sci. USA 105, 11737-11741 (2008).

9. Cheong, R., Rhee, A., Wang, C. J., Nemenman, I. \& Levchenko, A. Information transduction capacity of noisy biochemical signaling networks. Science 334, 354-358 (2011).

10. Uda, S. et al. Robustness and compensation of information transmission of signaling pathways. Science 341, 558-561 (2013).

11. Govern, C. C. \& ten Wolde, P. R. Optimal resource allocation in cellular sensing systems. Proc. Natl Acad. Sci. USA 111, 17486-17491 (2014).

12. Barkai, N. \& Leibler, S. Robustness in simple biochemical networks. Nature 387, 913-917 (1997). 
13. Alon, U., Surette, M. G., Barkai, N. \& Leibler, S. Robustness in bacterial chemotaxis. Nature 397, 168-171 (1999).

14. Tu, Y., Shimizu, T. S. \& Berg, H. C. Modeling the chemotactic response of Escherichia coli to time-varying stimuli. Proc. Natl Acad. Sci. USA 105, 1485514860 (2008).

15. Shimizu, T. S., Tu, Y. \& Berg, H. C. A modular gradient-sensing network for chemotaxis in Escherichia coli revealed by responses to time-varying stimuli. Mol. Syst. Biol. 6, 382 (2010).

16. Lan, G., Sartori, P., Neumann, S., Sourjik, V. \& Tu, Y. The energy-speedaccuracy trade-off in sensory adaptation. Nat. Phys. 8, 422-428 (2012).

17. Schreiber, T. Measuring information transfer. Phys. Rev. Lett. 85, 461 (2000).

18. Vicente, R., Wibral, M., Lindner, M. \& Pipa, G. Transfer entropy - a model-free measure of effective connectivity for the neurosciences. J. Comput. Neurosci. 30, 45-67 (2011).

19. Bauer, M., Cox, J. W., Caveness, M. H., Downs, J. J. \& Thornhill, N. F. Finding the direction of disturbance propagation in a chemical process using transfer entropy. IEEE Trans. Control Syst. Technol. 15, 12-21 (2007).

20. Lungarella, M. \& Sporns, O. Mapping information flow in sensorimotor networks. PLoS Comput. Biol 2, e144 (2006).

21. Massey, J. L. Causality, feedback and directed information. Proc. Int. Symp. Inf. Theory Appl. 303-305 (1990).

22. Shannon, C. E. A mathematical theory of communication. Bell Syst. Tech. J. 27, 379 (1948).

23. Cover, T. M. \& Thomas, J. A. Element of Information Theory (John Wiley and Sons, 1991).

24. Ito, S. \& Sagawa, T. Information thermodynamics on causal networks. Phys. Rev. Lett. 111, 180603 (2013).

25. Maxwell, J. C. Theory of Heat (Appleton, 1871).

26. Szilard, L. On the decrease of entropy in a thermodynamic system by the intervention of intelligent beings. Z. Phys. 53, 840-856 (1929).

27. Leff, H. S. \& Rex, A. F. (eds) Maxwell's Demon 2: Entropy, Classical and Quantum Information, Computing (Princeton University Press, 2003).

28. Sekimoto, K. Stochastic Energetics (Springer, 2010).

29. Seifert, U. Stochastic thermodynamics, fluctuation theorems and molecular machines. Rep. Prog. Phys. 75, 126001 (2012).

30. Allahverdyan, A. E., Janzing, D. \& Mahler, G. Thermodynamic efficiency of information and heat flow. J. Stat. Mech. P09011 (2009).

31. Sagawa, T. \& Ueda, M. Generalized Jarzynski equality under nonequilibrium feedback control. Phys. Rev. Lett. 104, 090602 (2010).

32. Toyabe, S., Sagawa, T., Ueda, M., Muneyuki, E. \& Sano, M. Experimental demonstration of information-to-energy conversion and validation of the generalized Jarzynski equality. Nat. Phys. 6, 988-992 (2010).

33. Horowitz, J. M. \& Vaikuntanathan, S. Nonequilibrium detailed fluctuation theorem for repeated discrete feedback. Phys. Rev. E 82, 061120 (2010).

34. Fujitani, Y. \& Suzuki, H. Jarzynski equality modified in the linear feedback system. J. Phys. Soc. Jpn. 79, $104003-104007$ (2010)

35. Horowitz, J. M. \& Parrondo, J. M. Thermodynamic reversibility in feedback processes. Euro. Phys. Lett. 95, 10005 (2011).

36. Ito, S. \& Sano, M. Effects of error on fluctuations under feedback control. Phys. Rev. E 84, 021123 (2011).

37. Sagawa, T. \& Ueda, M. Fluctuation theorem with information exchange: role of correlations in stochastic thermodynamics. Phys. Rev. Lett. 109, 180602 (2012).

38. Kundu, A. Nonequilibrium fluctuation theorem for systems under discrete and continuous feedback control. Phys. Rev. E 86, 021107 (2012).

39. Mandal, D. \& Jarzynski, C. Work and information processing in a solvable model of Maxwell's demon. Proc. Natl Acad. Sci. USA 109, 1164111645 (2012).

40. Bérut, A. et al. Experimental verification of Landauer's principle linking information and thermodynamics. Nature 483, 187189 (2012).

41. Hartich, D., Barato, A. C. \& Seifert, U. Stochastic thermodynamics of bipartite systems: transfer entropy inequalities and a Maxwell's demon interpretation. J. Stat. Mech. P02016 (2014).

42. Munakata, T. \& Rosinberg, M. L. Entropy production and fluctuation theorems for Langevin processes under continuous non-Markovian feedback control. Phys. Rev. Lett. 112, 180601 (2014).
43. Horowitz, J. M. \& Esposito, M. Thermodynamics with continuous information flow. Phys. Rev. X 4, 031015 (2014).

44. Barato, A. C., Hartich, D. \& Seifert, U. Efficiency of celluler information processing. New J. Phys. 16, 103024 (2014).

45. Sartori, P., Granger, L., Lee, C. F. \& Horowitz, J. M. Thermodynamic costs of information processing in sensory adaption. PLoS Compt. Biol. 10, e1003974 (2014).

46. Lang, A. H., Fisher, C. K., Mora, T. \& Mehta, P. Thermodynamics of statistical inference by cells. Phys. Rev. Lett. 113, 148103 (2014).

47. Horowitz, J. M. \& Sandberg, H. Second-law-like inequalities with information and their interpretations. New. J. Phys. 16, 125007 (2014).

48. Shiraishi, N. \& Sagawa, T. Fluctuation theorem for partially masked nonequilibrium dynamics. Phys. Rev. E 91, 012130 (2015).

49. Sartori, P. \& Tu, Y. Noise filtering strategies in adaptive biochemical signaling networks. J. Stat. Phys. 142, 1206-1217 (2011)

50. Kaiser, A. \& Schreiber, T. Information transfer in continuous processes. Physica D 166, 43-62 (2002).

51. Hlavackova-Schindler, K., Palus, M., Vejmelka, M. \& Bhattacharya, J. Causality detection based on information-theoretic approaches in time series analysis. Phys. Rep. 441, 1-46 (2007).

52. Barnett, L., Barrett, A. B. \& Seth, A. K. Granger causality and transfer entropy are equivalent for Gaussian variables. Phys. Rev. Lett. 103, 238701 (2009).

53. Collin, D. et al. Verification of the Crooks fluctuation theorem and recovery of RNA folding free energies. Nature 437, 231-234 (2005).

54. Ritort, F. Single-molecule experiments in biological physics: methods and applications. J. Phys. Condens. Matter 18, R531 (2006).

55. Toyabe, S. et al. Nonequilibrium energetics of a single F1-ATPase molecule. Phys. Rev. Lett. 104, 198103 (2010).

56. Hayashi, K., Ueno, H., Iino, R. \& Noji, H. Fluctuation theorem applied to F 1ATPase. Phys. Rev. Lett. 104, 218103 (2010).

\section{Acknowledgements}

We are grateful to S.-I. Sasa, U. Seifert, M. L. Rosinberg, N. Shiraishi, K. Kawaguchi, H. Tajima, A.C. Barato, D. Hartich and M. Sano for their valuable discussions. This work was supported by the Grants-in-Aid for JSPS Fellows (grant no. $24 \cdot 8593$ ), by JSPS KAKENHI grant numbers 25800217 and 22340114, by KAKENHI no. 25103003 'Fluctuation \& Structure' and by Platform for Dynamic Approaches to Living System from MEXT, Japan.

\section{Author contributions}

S.I. mainly constructed the theory, carried out the analytical and numerical calculations, and wrote the paper. T.S. also constructed the theory and wrote the paper. Both authors discussed the results at the all stages.

\section{Additional information}

Supplementary Information accompanies this paper at http://www.nature.com/ naturecommunications

Competing financial interests: The authors declare no competing financial interests.

Reprints and permission information is available online at http://npg.nature.com/ reprintsandpermissions/

How to cite this article: Ito, S. and Sagawa, T. Maxwell's demon in biochemical signal transduction with feedback loop. Nat. Commun. 6:7498 doi: 10.1038/ncomms8498 (2015).

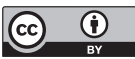

This work is licensed under a Creative Commons Attribution 4.0 International License. The images or other third party material in this article are included in the article's Creative Commons license, unless indicated otherwise in the credit line; if the material is not included under the Creative Commons license, users will need to obtain permission from the license holder to reproduce the material. To view a copy of this license, visit http://creativecommons.org/licenses/by/4.0/ 